\title{
Closed form Solutions of New Fifth Order Nonlinear Equation and New Generalized Fifth Order Nonlinear Equation via the Enhanced ( $\left.G^{\prime} / G\right)$-expansion Method
}

\author{
A K M Kazi Sazzad Hossain ${ }^{1 *}$ and Md. Ali Akbar ${ }^{2}$ \\ ${ }^{1}$ Department of Mathematics, Begum Rokeya University, Bangladesh \\ ${ }^{2}$ Department of Applied Mathematics, University of Rajshahi, Bangladesh \\ Submission: October 2, 2017; Published: December 08, 2017 \\ *Corresponding author: A K M Kazi Sazzad Hossain, Department of Mathematics, Begum Rokeya University, Rangpur, Bangladesh, Tel: 8.80171E+12; \\ Email: kazi_bru@yahoo.com
}

\begin{abstract}
Closed form solutions of nonlinear evolution equations (NLEEs) are very imperative in order to better understand the inner mechanism and complexity of complex physical phenomena. The enhanced $\left(G^{\prime} / G\right)$-expansion method is a effectual and proficient mathematical tool which can be used to discover the closed form solutions of NLEEs arising in mathematical physics, applied mathematics and engineering. In this article, the enhanced $\left(G^{\prime} / G\right)$-expansion method is recommended and carry out to investigate the closed form solutions of the new fifth order non-linear equation and the new generalized fifth order non-linear equation. The performance of this method is reliable, proficient and possible to obtain a lot of new exact solutions than the existing other methods.

Keywords: The enhanced $\left(G^{\prime} / G\right)$-expansion method; New fifth order nonlinear equation; New generalized fifth order nonlinear equation; Nonlinear evolution equations (NLEEs); Closed form solutions
\end{abstract}

\section{Introduction}

Closed form solutions of nonlinear evolution equations (NLEEs) are getting importance to study of complex phenomena in the field of science and engineering. NLEEs are frequently appear in various fields, such as plasma physics, geophysics, nuclear physics, biomathematics optical fibers, biomechanics, gas dynamics, chemical reactions, geochemistry etc. Closed form solutions of NLEEs and its graphical representation reveal the inner mechanism of complex nonlinear phenomena. Therefore, it is a urgent issue and very important to search for more closed form solutions to NLEEs in order to better realization of the structure of nonlinear phenomena. But till now there is no distinctive method to inspect all kinds NLEEs. As a result diverse groups of mathematicians, physicist and engineers have been working vigorously to develop effective methods for which to solve all NLEEs.

For this reason, in the recent years there has been considerable progress in the development of finding effective methods to search exact solution, such as the nonlinear transform method [1], the first integration method [2], the F-expansion method [3], the Exp-function method [4-6], the Jacobi-elliptic function method [7,8], the Darboux transformation method [9], the complex hyperbolic function method $[10,11]$, the auxiliary equation method [12], the Adomian decomposition method [13], the functional variable method [14], the sine cosine method [15], the Painleve expansion method [16], the $\exp (-\Phi(\eta))$ expansion method $[17,18]$, the variational method $[19,20]$, the simplified Hirota's method [21], the sine-cosine method [22], the Kudryashov method [23], the extended direct algebraic method $[24,25]$, the modified simple equation method [26-30], the Lie group symmetry method [31], the $\left(G^{\prime} / G\right)$-expansion method [32$36]$, the enhanced $\left(G^{\prime} / G\right)$-expansion method $[37,38]$, the improve $\left(G^{\prime} / G\right)$-expansion method, etc.

The recently developed the enhanced $\left(G^{\prime} / G\right)$-expansion method is getting popularity in use because of its directly advanced calculation procedure and there is possible to obtain large number of solution. The objective of this article is to introduce and make use of the enhanced $\left(G^{\prime} / G\right)$-expansion method to extract fresh and further general exact traveling wave solutions to the new fifth order nonlinear equation and new generalized fifth order nonlinear equation. The rest of the article is arranged as follows: In Section 2, enhanced $\left(G^{\prime} / G\right)$-expansion method is discussed. In Section 3, the enhanced $\left(G^{\prime} / G\right)$-expansion method is applied to examine the NLEEs point out above. In Section 4, 
results and physical explanations and In Section 5 conclusions are provided.

\section{Algorithm of the enhanced $\left(G^{\prime} / G\right)$-expansion}

In this section, we explore the enhanced $\left(G^{\prime} / G\right)$-expansion method for finding traveling wave solutions to NLEEs. Let us consider a nonlinear evolution equation in two independent variables $\mathrm{x}$ and $\mathrm{t}$ in the form:

$$
R\left(u, u_{t}, u_{x}, u_{t t}, u_{x x}, u_{x t}, \cdots\right)=0,
$$

Where $u=u(x, t)$ is an unknown function of $x$ and $t$ and $R$ is a polynomial of $u(x, t)$ and its partial derivatives which contains the highest degree nonlinear terms. The essential steps concerning this method are offered in the following:

Step 1: Initiating a compound variable $\xi$ with the combination of real variables $x$ and $t$,

$$
u(x, t)=u(\xi), \xi=x \pm \omega t
$$

Where, specify the speed of the traveling wave.

The traveling wave transformations (2.2) permit us in dropping Eq. (2.1) to an ordinary differential equation (ODE) for $u=u(\xi)$ in the form:

$$
S\left(u, u^{\prime}, u^{\prime \prime}, u^{\prime \prime \prime} \ldots\right)=0,(2.3)
$$

Where, $\mathrm{S}$ is a polynomial in $u(\xi)$ and its derivatives with respect to $\xi$.

Step 2: The solution of Eq. (2.3) can be expressed in the following form:

$$
u(\xi)=\sum_{i=-n}^{n}\left(\frac{a_{i}\left(G^{\prime} / G\right)^{i}}{\left(1+\lambda\left(G^{\prime} / G\right)\right)^{i}}+b_{i}\left(G^{\prime} / G\right)^{i-1} \sqrt{\sigma\left(1+\frac{\left(G^{\prime} / G\right)^{2}}{\mu}\right)}\right)
$$

Where, $a_{i}, b_{i}(-n \leq i \leq n ; n \in N)$ are constants to be determined later, $\sigma= \pm 1, \quad \mu \neq 0$ and $G=G(\xi)$ satisfies the equation

$$
G^{\prime \prime}+\mu G=0 \text {. (2.5) }
$$

Step 3: Taking homogeneous balance between the highest order linear term and the nonlinear terms of the highest degree which appear in Eq. (2.3), we obtain the limiting value $n$.

Step 4: Substituting (2.4) into (2.3) together with (2.5) and then accumulating all terms of same powers of $\left(G^{\prime} / G\right)^{i}$ and $\left(G^{\prime} / G\right)^{j} \sqrt{\sigma\left(1+\frac{\left(G^{\prime} / G\right)^{2}}{\mu}\right)}$ and setting each coefficient to zero yields a system of algebraic equations for $a_{i}, b_{i}(-n \leq i \leq n ; n \in N), \lambda$ and $\omega$. Solving this system of equations supplies the values of the unknown parameters.

Step 5: From the general solution of equation (2.5), we obtain

$$
\begin{aligned}
& \text { When, } \mu<0 \text {, } \\
& \frac{G^{\prime}}{G}=\sqrt{-\mu} \tanh \left(\xi_{0}+\sqrt{-\mu} \xi\right) \text {, }
\end{aligned}
$$

And BBOAJ.2017.04.555631.

$$
\frac{G^{\prime}}{G}=\sqrt{-\mu} \operatorname{coth}\left(\xi_{0}+\sqrt{-\mu} \xi\right)
$$

Again when $\mu>0$,

$$
\frac{G^{\prime}}{G}=\sqrt{\mu} \tan \left(\xi_{0}-\sqrt{\mu} \xi\right),
$$

And

$$
\frac{G^{\prime}}{G}=\sqrt{\mu} \cot \left(\xi_{0}+\sqrt{\mu} \xi\right)
$$

Where, $\xi_{0}$ is an arbitrary constant. At last, substituting $a_{i}, b_{i}(-n \leq i \leq n ; n \in N), \lambda$ and $\omega$ and solutions (2.6)-(2.9) into (2.4), we obtain more general and some fresh traveling wave solutions of (2.1).

\section{Applications of the method}

In this section, we inspect the closed form solutions of the new fifth order non-linear equation and new generalized fifth order non-linear equation with the help of the enhanced $\left(G^{\prime} / G\right)$-expansion method.

Example 1: In this subsection, we will use the enhanced $\left(G^{\prime} / G\right)$-expansion method search for the exact solution to the following new fifth order non-linear equation of the form [21]

$$
u_{t t t}-u_{t x x x x}-4\left(u_{x} u_{t}\right)_{x x}-4\left(u_{x} u_{x t}\right)_{x}=0
$$

The equation (3.1) transfer to ODE in the following form using wave transformation (2.2)

$$
-\omega^{3} u^{\prime \prime \prime}+\omega u^{(v)}+4 \omega\left(u^{\prime} u^{\prime}\right)^{\prime \prime}+4 \omega\left(u^{\prime} u^{\prime \prime}\right)^{\prime}=0 \text {. }
$$

Integrating (3.2) with respect to twice and taking integration constant to zero, we get

$$
u^{\prime \prime \prime}+6 u^{\prime 2}-\omega^{2} u^{\prime}=0 \text {. (3.3) }
$$

balancing the highest-order derivative term $u^{\prime \prime \prime}$ and the highest-order nonlinear term $u^{\prime 2}$ yields $n=1$. Thus, the solution structure of Eq. (3.3) becomes

$$
\begin{aligned}
& u(\xi)=a_{0}+\frac{a_{1}\left(\frac{G^{\prime}}{G}\right)}{1+\lambda\left(\frac{G^{\prime}}{G}\right)^{\prime}}+\frac{a_{-1}\left(1+\lambda\left(\frac{G^{\prime}}{G}\right)\right)}{\left(\frac{G^{\prime}}{G}\right)}+b_{0}\left(\frac{G^{\prime}}{G}\right)^{-1} \sigma\left(1+\frac{\left(\frac{G}{G}\right)^{2}}{\mu}\right)+b_{1} \sigma\left(1+\frac{\left(\frac{G}{G}\right)^{2}}{\mu}\right) \\
& +b_{-1}\left(\frac{G^{\prime}}{G}\right)^{-2} \sigma\left(1+\frac{\left(\frac{G^{\prime}}{G}\right)^{2}}{\mu}\right)
\end{aligned}
$$

Where, $G=G(\xi)$ satisfies Eq. (2.5).

Substituting (3.4) with the equation (2.5) into equation (3.3), we attain a polynomial $\left(G^{\prime} / G\right)^{i}$ of and $\left(_{\left(G^{\prime} / G\right)^{\prime}} \sqrt{\sigma\left(1+\frac{\left(G^{\prime} / G\right)^{2}}{\mu}\right)}\right.$. From this polynomial we get the coefficients $\left(G^{\prime} / G\right)^{i}$ of and $\left.{ }^{\prime} G^{\prime} / G\right)^{\prime} \sqrt{\sigma\left(1+\frac{\left(G^{\prime} / G^{2}\right.}{\mu}\right)}$. Equating them to zero, we achieve an over-determined system that contains thirty algebraic equations (for simplicity we skip to display them). Solving this system of algebraic equation, we get

Set 1: $\omega= \pm 2 \sqrt{-\mu}, \lambda=\lambda, a_{-1}=0, a_{0}=a_{0}, a_{1}=1+\mu \lambda^{2}, b_{-1}=0, b_{0}=0, b_{1}=0$. 
Set 2: $\omega= \pm \sqrt{-\mu}, \lambda=0, a_{-1}=0, a_{0}=a_{0}, a_{1}=\frac{1}{2}, b_{-1}=0, b_{0}=0, b_{1}= \pm \frac{\sqrt{\mu}}{2 \sqrt{\sigma}}$.

Set 3: $\omega= \pm 2 \sqrt{-\mu}, \lambda=\lambda, a_{-1}=-\mu, a_{0}=a_{0}, a_{1}=0, b_{-1}=0, b_{0}=0, b_{1}=0$

Set 4: $\omega= \pm 4 \sqrt{-\mu}, \lambda=0, a_{-1}=-\mu, a_{0}=a_{0}, a_{1}=1, b_{-1}=0, b_{0}=0, b_{1}=0$.

Set 5: $\omega= \pm \sqrt{-\mu}, \lambda=\lambda, a_{-1}=-\frac{\mu}{2}, a_{0}=a_{0}, a_{1}=0, b_{-1}=0, b_{0}= \pm \frac{\mu}{2 \sqrt{\sigma}}, b_{1}=0$.

Now substituting solution set 1-5 with equation (2.5) into equation (3.4), we get sufficient traveling wave solution to Eq. (3.1) as follows:

When, $<$, we get the hyperbolic solution,

Profile-1: $u_{1}(\xi)=a_{0}+\left(1+\mu \lambda^{2}\right) \frac{\sqrt{-\mu} \tanh \left(\xi_{0}+\sqrt{-\mu} \xi\right)}{\left(1+\lambda \sqrt{-\mu} \tanh \left(\xi_{0}+\sqrt{-\mu} \xi\right)\right)}$

$$
u_{1}(\xi)=a_{0}+\left(1+\mu \lambda^{2}\right) \frac{\sqrt{-\mu} \tanh \left(\xi_{0}+\sqrt{-\mu} \xi\right)}{\left(1+\lambda \sqrt{-\mu} \tanh \left(\xi_{0}+\sqrt{-\mu} \xi\right)\right)}
$$

Where $\xi=x \pm 2 \sqrt{-\mu} t$,

Profile -2:

$u_{3}(\xi)=a_{0}+\frac{1}{2} \sqrt{-\mu} \tanh \left(\xi_{0}+\sqrt{-\mu} \xi\right) \pm \frac{1}{2} \sqrt{\mu\left(1-\left(\tanh \left(\xi_{0}+\sqrt{-\mu} \xi\right)\right)^{2}\right)}$

$u_{4}(\xi)=a_{0}+\frac{1}{2} \sqrt{-\mu} \operatorname{coth}\left(\xi_{0}+\sqrt{-\mu} \xi\right) \pm \frac{1}{2} \sqrt{\mu\left(1-\left(\operatorname{coth}\left(\xi_{0}+\sqrt{-\mu} \xi\right)\right)^{2}\right.}$

Where $\xi=x \pm \sqrt{-\mu} t$

Profile-3:

$$
u_{5}(\xi)=a_{0}-\left(\lambda \mu+\sqrt{-\mu} \operatorname{coth}\left(\xi_{0}+\sqrt{-\mu} \xi\right)\right) \text { llioxet (3.9) }
$$

$u_{6}(\xi)=a_{0}-\left(\lambda \mu+\sqrt{-\mu} \tanh \left(\xi_{0}+\sqrt{-\mu} \xi\right)\right)$ (3.10)

Where $\xi=x \pm 2 \sqrt{-\mu} t$

\section{Profile-4:}

$u_{7}(\xi)=a_{0} \pm \sqrt{-\mu}\left(\tanh \left(\xi_{0}+\sqrt{-\mu} \xi\right)-\operatorname{coth}\left(\xi_{0}+\sqrt{-\mu} \xi\right)\right)$

Where $\xi=x \pm 4 \sqrt{-\mu} t$

\section{Profile-5:}

$u_{8}(\xi)=a_{0}-\frac{\sqrt{-\mu}}{2} \operatorname{coth}\left(\xi_{0}+\sqrt{-\mu} \xi\right)\left(\left(1+\lambda \sqrt{-\mu} \tanh \left(\xi_{0}+\sqrt{-\mu} \xi\right)\right) \mp \sqrt{\left(1-\left(\tanh \left(\xi_{0}+\sqrt{-\mu} \xi\right)\right)^{2}\right)}\right)$

$u_{9}(\xi)=a_{0}-\frac{\sqrt{-\mu}}{2} \tanh \left(\xi_{0}+\sqrt{-\mu} \xi\right)\left(\left(1+\lambda \sqrt{-\mu} \operatorname{coth}\left(\xi_{0}+\sqrt{-\mu} \xi\right)\right) \mp \sqrt{\left(1-\left(\operatorname{coth}\left(\xi_{0}+\sqrt{-\mu} \xi\right)\right)^{2}\right)}\right)$

$=(3.13)$

Where $\xi=x \pm \sqrt{-\mu t}$

Again, for $\mu>0$, we get the following trigonometric solution:

Profile-6:

$u_{10}(\xi)=a_{0}+\left(1+\mu \lambda^{2}\right) \frac{\sqrt{\mu} \tan \left(\xi_{0}-\sqrt{\mu} \xi\right)}{\left(1+\lambda \sqrt{\mu} \tan \left(\xi_{0}-\sqrt{\mu} \xi\right)\right)}$ $u_{11}(\xi)=a_{0}+\left(1+\mu \lambda^{2}\right) \frac{\sqrt{\mu} \cot \left(\xi_{0}+\right.}{(1+\lambda \sqrt{\mu} \cot (\xi}$

Where $\xi=x \pm 2 \sqrt{-\mu} t$

Profile-7:

$u_{12}(\xi)=a_{0}+\frac{\sqrt{\mu}}{2}\left(\tan \left(\xi_{0}-\sqrt{\mu} \xi\right) \pm \sqrt{\left(1+\left(\tan \left(\xi_{0}-\sqrt{\mu} \xi\right)\right)^{2}\right)}\right)$

$u_{13}(\xi)=a_{0}+\frac{\sqrt{\mu}}{2}\left(\cot \left(\xi_{0}+\sqrt{\mu} \xi\right) \pm \sqrt{\left(1+\left(\cot \left(\xi_{0}+\sqrt{\mu} \xi\right)\right)^{2}\right)}\right)$

Where nnw $\xi=x \pm \sqrt{-\mu t}$

Profile-8:

$u_{14}(\xi)=a_{0}-\left(\lambda \mu+\sqrt{\mu} \cot \left(\xi_{0}-\sqrt{\mu} \xi\right)\right) 3.18$

$u_{15}(\xi)=a_{0}-\left(\lambda \mu+\sqrt{\mu} \tan \left(\xi_{0}+\sqrt{\mu} \xi\right)\right)$

Where, $\xi=x \pm 2 \sqrt{-\mu} t$

Profile-9:

$u_{16}(\xi)=a_{0}+\sqrt{\mu}\left(\tan \left(\xi_{0}-\sqrt{\mu} \xi\right)-\cot \left(\xi_{0}-\sqrt{\mu} \xi\right)\right)$

$u_{17}(\xi)=a_{0}+\sqrt{\mu}\left(\cot \left(\xi_{0}+\sqrt{\mu} \xi\right)-\tan \left(\xi_{0}+\sqrt{\mu} \xi\right)\right)$

Where, $\xi=x \pm 4 \sqrt{-\mu t}$

Profile-10:

$u_{18}(\xi)=a_{0}-\frac{\sqrt{\mu}}{2} \cot \left(\xi_{0}-\sqrt{\mu} \xi\right)\left(\left(1+\lambda \sqrt{\mu} \tan \left(\xi_{0}-\sqrt{\mu} \xi\right)\right) \mp \sqrt{\left(1+\left(\tan \left(\xi_{0}-\sqrt{\mu} \xi\right)\right)^{2}\right)}\right)$

$\left.u_{19}(\xi)=a_{0}-\frac{\sqrt{\mu}}{2} \tan \left(\xi_{0}+\sqrt{-\mu} \xi\right) \tan \left(\xi_{0}+\sqrt{-\mu} \xi\right)\left(\left(1+\lambda \sqrt{\mu} \cot \left(\xi_{0}+\sqrt{\mu} \xi\right)\right) \mp \sqrt{\left(1+\left(\cot \left(\xi_{0}+\sqrt{\mu} \xi\right)\right)^{2}\right.}\right)\right)$

Where, $\xi=x \pm \sqrt{-\mu} t$

Example 2: In this subsection, we will apply the given method in section 2 for the exact solution and then the solitary wave solution to the following generalized new fifth order nonlinear equation of the form [21]

$u_{t t t}-u_{t x x x x}-\alpha\left(u_{x} u_{t}\right)_{x x}-\beta\left(u_{x} u_{x t}\right)_{x}=0$

Where, $\alpha$ and $\beta$ are constant.

The traveling wave transformation $u(x, t)=u(\xi), \xi=k x-\omega t$, switches (3.24) to the ODE in the form

$-\omega^{3} u^{\prime \prime \prime}+\omega k^{4} u^{(v)}+\alpha \omega k^{3}\left(u^{\prime} u^{\prime}\right)^{\prime \prime}+\beta \omega k^{3}\left(u^{\prime} u^{\prime \prime}\right)^{\prime}=0$

Integrating (3.2) with respect to $\xi$ twice and taking integration constant to zero, we attain

$k^{4} u^{\prime \prime \prime}+k^{3}(\alpha+\beta / 2) u^{\prime 2}-\omega^{2} u^{\prime}=0$ (3.26) 
Balancing the highest-order derivative term $u^{\prime \prime \prime}$ and the highest-order nonlinear term $u^{2}$, yields $n=1$.

Thus, the solution structure of Eq. (3.26) becomes

$u(\xi)=a_{0}+\frac{a_{1}\left(\frac{G^{\prime}}{G}\right)}{1+\lambda\left(\frac{G^{\prime}}{G}\right)}+\frac{a_{-1}\left(1+\lambda\left(\frac{G^{\prime}}{G}\right)\right)}{\left(\frac{G^{\prime}}{G}\right)}+b_{0}\left(\frac{G^{\prime}}{G}\right)^{-1} \sigma\left(1+\frac{\left(\frac{G^{\prime}}{G}\right)^{2}}{\mu}\right)+b_{1} \sigma\left(1+\frac{\left(\frac{G^{\prime}}{G}\right)^{2}}{\mu}\right)+b_{-1}\left(\frac{G^{\prime}}{G}\right)^{-2} \sigma\left(1+\frac{\left(\frac{G^{\prime}}{G}\right)^{2}}{\mu}\right)$

Where, $G=G(\xi)$ satisfies Eq. (2.5).

Replacing (3.27) with the equation (2.5) into equation (3.26), we achieve a polynomial of $\left(G^{\prime} / G\right)^{i}$ and $\left(G^{\prime} / G\right)^{\prime} \sqrt{\sigma\left(1+\frac{\left(G^{\prime} / G\right)^{2}}{\mu}\right)}$. Equating the coefficient of these to zero, we achieve a system of algebraic equation which on solving, we get

Set 1: $\omega= \pm 2 k^{2} \sqrt{-\mu}, \lambda=\lambda, a_{-1}=0, a_{0}=a_{0}, a_{1}=\frac{12 k\left(1+\mu \lambda^{2}\right)}{(2 \alpha+\beta)}, b_{-1}=0, b_{0}=0, b_{1}=0$.

Set 2: $\omega= \pm k^{2} \sqrt{-\mu}, \lambda=0, a_{-1}=0, a_{0}=a_{0}, a_{1}=\frac{6 k}{(2 \alpha+\beta)}, b_{-1}=b_{0}=0, b_{1}= \pm \frac{6 k \sqrt{\mu}}{(2 \alpha+\beta) \sqrt{\sigma}}$. .

Set 3: $\omega= \pm 2 k^{2} \sqrt{-\mu}, \lambda=\lambda, a_{-1}=-\frac{12 k \mu}{(2 \alpha+\beta)}, a_{0}=a_{0}, a_{1}=0, b_{-1}=0, b_{0}=0, b_{1}=0$

Set 4: $\omega= \pm 4 k^{2} \sqrt{-\mu}, \lambda=0, a_{-1}=-\frac{12 k \mu}{(2 \alpha+\beta)}, a_{0}=a_{0}, a_{1}=\frac{12 k}{(2 \alpha+\beta)}, b_{-1}=b_{0}=0, b_{1}=0$.

Set 5: $\omega= \pm 2 k^{2} \sqrt{-\mu}, \lambda=\lambda, a_{-1}=-\frac{6 k \mu}{(2 \alpha+\beta)}, a_{0}=a_{0}, a_{1}=b_{-1}=b_{1}=0, b_{0}= \pm \frac{6 k \mu}{(2 \alpha+\beta) \sqrt{\sigma}}$.

Now setting solution set 1-5 with equation (2.5) into equation (3.27), we get adequate traveling wave solution to Eq. (3.24) as follows:

When, $\mu<0$, we get the hyperbolic solution,

Type-1:

$u_{2_{1}}(\xi)=a_{0}+\frac{12 k\left(1+\mu \lambda^{2}\right)}{(2 \alpha+\beta)} \frac{\sqrt{-\mu} \tanh \left(\xi_{0}+\sqrt{-\mu} \xi\right)}{\left(1+\lambda \sqrt{-\mu} \tanh \left(\xi_{0}+\sqrt{-\mu} \xi\right)\right)}$

$u_{2_{2}}(\xi)=a_{0}+\frac{12 k\left(1+\mu \lambda^{2}\right)}{(2 \alpha+\beta)} \frac{\sqrt{-\mu} \operatorname{coth}\left(\xi_{0}+\sqrt{-\mu} \xi\right)}{\left(1+\lambda \sqrt{-\mu} \operatorname{coth}\left(\xi_{0}+\sqrt{-\mu} \xi\right)\right)}$

Where, $\xi=x \pm 2 k^{2} \sqrt{-\mu} t$

Type -2:

$u_{2_{3}}(\xi)=a_{0}+\frac{6 k}{(2 \alpha+\beta)}\left(\sqrt{-\mu} \tanh \left(\xi_{0}+\sqrt{-\mu} \xi\right) \pm \sqrt{\mu\left(1-\left(\tanh \left(\xi_{0}+\sqrt{-\mu} \xi\right)\right)^{2}\right)}\right)(3.30)$ (3.30)

$u_{2_{3}}(\xi)=a_{0}+\frac{6 k}{(2 \alpha+\beta)}\left(\sqrt{-\mu} \tanh \left(\xi_{0}+\sqrt{-\mu} \xi\right) \pm \sqrt{\mu\left(1-\left(\tanh \left(\xi_{0}+\sqrt{-\mu} \xi\right)\right)^{2}\right)}\right)$ (3.30) (3.31)

Where, $\xi=x \pm k^{2} \sqrt{-\mu t}$

Type-3:

$u_{2_{5}}(\xi)=a_{0}-\frac{12 k}{(2 \alpha+\beta)}\left(\lambda \mu+\sqrt{-\mu} \operatorname{coth}\left(\xi_{0}+\sqrt{-\mu} \xi\right)\right)$

$u_{2_{6}}(\xi)=a_{0}-\frac{12 k}{(2 \alpha+\beta)}\left(\lambda \mu+\sqrt{-\mu} \tanh \left(\xi_{0}+\sqrt{-\mu} \xi\right)\right)$

Where, $\xi=x \pm 2 k^{2} \sqrt{-\mu}$,
Type-4:

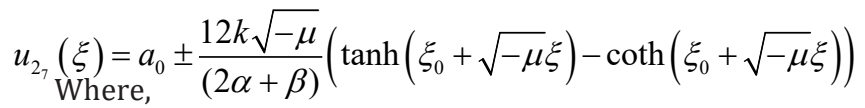

Type-5:

$u_{2 \mathrm{z}}(\xi)=a_{0}-\frac{6 k \sqrt{-\mu}}{(2 \alpha+\beta)} \operatorname{coth}\left(\xi_{0}+\sqrt{-\mu} \xi\right)\left(\left(1+\lambda \sqrt{-\mu} \tanh \left(\xi_{0}+\sqrt{-\mu} \xi\right)\right) \mp \sqrt{\left(1-\left(\tanh \left(\xi_{0}+\sqrt{-\mu} \xi\right)\right)^{2}\right)}\right)$

$u_{2,}(\xi)=a_{0}-\frac{6 k \sqrt{-\mu}}{(2 \alpha+\beta)} \tanh \left(\xi_{0}+\sqrt{-\mu} \xi\right)\left(\left(1+\lambda \sqrt{-\mu} \operatorname{coth}\left(\xi_{0}+\sqrt{-\mu} \xi\right)\right) \mp \sqrt{\left(1-\left(\operatorname{coth}\left(\xi_{0}+\sqrt{-\mu} \xi\right)\right)^{2}\right)}\right)$

Where, $\xi=x \pm k^{2} \sqrt{-\mu} t$

Again, for $\mu>0$, we get the following trigonometric solution:

Type-6:

$u_{2_{10}}(\xi)=a_{0}+\frac{12 k\left(1+\mu \lambda^{2}\right)}{(2 \alpha+\beta)} \frac{\sqrt{\mu} \tan \left(\xi_{0}-\sqrt{\mu} \xi\right)}{\left(1+\lambda \sqrt{\mu} \tan \left(\xi_{0}-\sqrt{\mu} \xi\right)\right)}$

$u_{2_{11}}(\xi)=a_{0}+\frac{12 k\left(1+\mu \lambda^{2}\right)}{(2 \alpha+\beta)} \frac{\sqrt{\mu} \cot \left(\xi_{0}+\sqrt{\mu} \xi\right)}{\left(1+\lambda \sqrt{\mu} \cot \left(\xi_{0}+\sqrt{\mu} \xi\right)\right)}$

Where, $\xi=x \pm 2 k^{2} \sqrt{-\mu} t$

Type-7:

$u_{212}(\xi)=a_{0}+\frac{6 k \sqrt{\mu}}{(2 \alpha+\beta)}\left(\tan \left(\xi_{0}-\sqrt{\mu} \xi\right) \pm \sqrt{\left(1+\left(\tan \left(\xi_{0}-\sqrt{\mu} \xi\right)\right)^{2}\right)}\right)$

$u_{2_{13}}(\xi)=a_{0}+\frac{6 k \sqrt{\mu}}{(2 \alpha+\beta)}\left(\cot \left(\xi_{0}+\sqrt{\mu} \xi\right) \pm \sqrt{\left(1+\left(\cot \left(\xi_{0}+\sqrt{\mu} \xi\right)\right)^{2}\right)}\right)$

Where, $\xi=x \pm k^{2} \sqrt{-\mu t}$

Type-8:

$u_{2_{14}}(\xi)=a_{0}-\frac{12 k}{(2 \alpha+\beta)}\left(\lambda \mu+\sqrt{\mu} \cot \left(\xi_{0}-\sqrt{\mu} \xi\right)\right)$

$u_{2_{15}}(\xi)=a_{0}-\frac{12 k}{(2 \alpha+\beta)}\left(\lambda \mu+\sqrt{\mu} \tan \left(\xi_{0}+\sqrt{\mu} \xi\right)\right)$

Where,

Type-9:

$u_{2_{16}}(\xi)=a_{0}+\frac{12 k \sqrt{\mu}}{(2 \alpha+\beta)}\left(\tan \left(\xi_{0}-\sqrt{\mu} \xi\right)-\cot \left(\xi_{0}-\sqrt{\mu} \xi\right)\right)$

$u_{2_{17}}(\xi)=a_{0}+\frac{12 k \sqrt{\mu}}{(2 \alpha+\beta)}\left(\cot \left(\xi_{0}+\sqrt{\mu} \xi\right)-\tan \left(\xi_{0}+\sqrt{\mu} \xi\right)\right)$

Where, $u_{217}(\xi)=a_{0}+\frac{12 k \sqrt{\mu}}{(2 \alpha+\beta)}\left(\cot \left(\xi_{0}+\sqrt{\mu} \xi\right)-\tan \left(\xi_{0}+\sqrt{\mu} \xi\right)\right)$

Type-10:

$\left.u_{2, \mathrm{~s}}=a_{0}+\frac{6 k \sqrt{\mu}}{(2 \alpha+\beta)} \cot \left(\xi_{0}-\sqrt{\mu} \xi\right)\left(\left(1+\lambda \sqrt{\mu} \tan \left(\xi_{0}-\sqrt{\mu} \xi\right)\right)+\sqrt{\left(1+\left(\tan \left(\xi_{0}-\sqrt{\mu} \xi\right)\right)^{2}\right.}\right)\right)$ 
$\left.u_{219}=a_{0}-\frac{6 k \sqrt{\mu}}{(2 \alpha+\beta)} \tan \left(\xi_{0}+\sqrt{\mu} \xi\right)\left(\left(1+\lambda \sqrt{\mu} \cot \left(\xi_{0}+\sqrt{\mu} \xi\right)\right) \mp \sqrt{\left(1+\left(\cot \left(\xi_{0}+\sqrt{\mu} \xi\right)\right)^{2}\right.}\right)\right)$ (3.46)

Where, $\xi=x \pm k^{2} \sqrt{-\mu t}$.

\section{Results and Physical Explanations}

In this section, we have discussed about the obtained solution of new fifth order non-linear equation and new generalized fifth order non-linear equation. As of the above solution, it has been noticed that $\sigma= \pm 1$ and $\mu \neq 0$. The negative values of $\mu$ gives the hyperbolic solutions $u_{1}(\xi)-u_{9}(\xi)$ of the new fifth order nonlinear equation through Profile 1 to 5 and positive values of $\mu$ gives, trigonometric solutions $u_{10}(\xi)-u_{19}(\xi)$ through Profile 6 to 10 . The solutions $u_{2}(\xi)$ and $u_{8}(\xi)$ demonstrate the nature of kink wave. Solutions $u_{1}(\xi), u_{5}(\xi)$ and $u_{7}(\xi)$ display the nature of singular kink wave. Moreover, solutions $u_{10}(\xi)-u_{19}(\xi)$ represent the character of periodic traveling wave. The solutions $u_{4}(\xi)$ and $u_{6}(\xi)$ show the nature of soliton solution where $u_{3}(\xi)$ and $u_{9}(\xi)$ express the singular solution. The graphical illustrations of some obtained solutions are given below. The Figure 1 represents the soliton solution $u_{4}(\xi)$ in (3.8) for $\mu=-1, \sigma=-1, \xi_{0}=5, a_{0}=3, \lambda=0$ within $-10 \leq x, t \leq 10$. Singular kink wave solution $u_{5}(\xi)$ for $\mu=-1, \xi_{0}=1, a_{0}=3, \lambda=2$ and kink shape wave solution of $u_{8}(\xi)$ for $\mu=-2, \sigma=1, \xi_{0}=3, a_{0}=2, \lambda=1$ within the interval $-10 \leq x, t \leq 10$ have been shown in Figure 2 and Figure 3 respectively. Periodic wave solution $u_{19}(\xi)$ in (3.23) for $\mu=1, \sigma=1, \xi_{0}=1, a_{0}=2, \lambda=1$ within the interval $-10 \leq x, t \leq 10$ and $-5 \leq t \leq 5$ are given by Figure 4 .

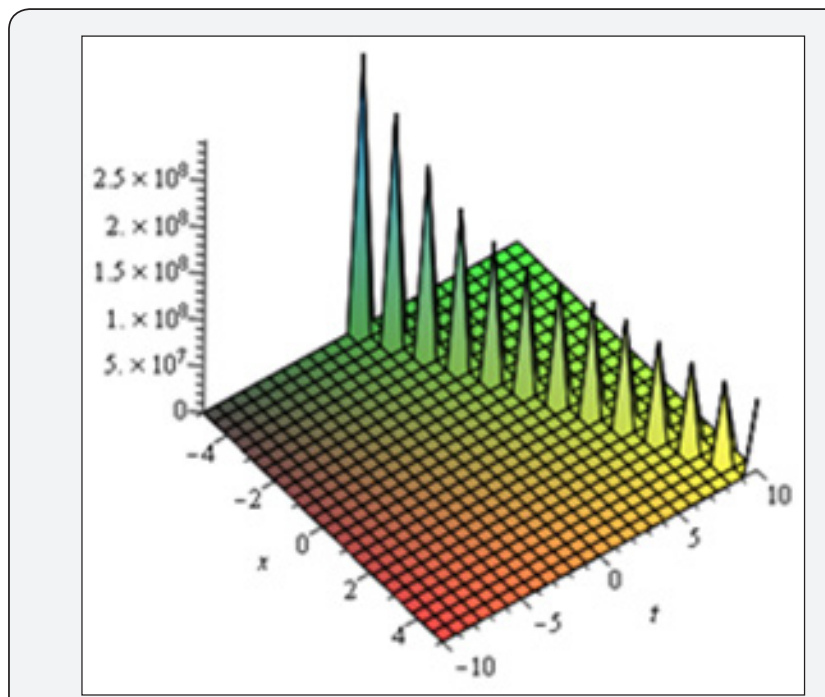

Figure 1: Soliton solution $u_{4}(\xi)$ in (3.8) for $\mu=-1, \sigma=-1, \xi_{0}=5, a_{0}=3, \lambda=0$.

Again from the solutions of the new generalized fifth order non-linear equation, it is observed that the negative values

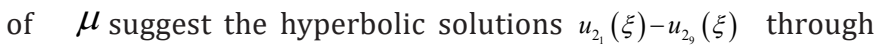
Type 1 to 5 and the positive values of $\mu$, recommend the trigonometric solutions $u_{2_{10}}(\xi)-u_{2_{19}}(\xi)$ through Type 6 to 10 .
The solutions $u_{2_{2}}(\xi)$ and $u_{2_{6}}(\xi)$ demonstrate the kink wave. The solutions $u_{2_{1}}(\xi), u_{2_{\xi}}(\xi), u_{2,}(\xi)$ and $u_{2_{8}}(\xi)$ are singular kink wave solution. Furthermore, the solutions $u_{2_{10}}(\xi)-u_{2_{19}}(\xi)$ are the periodic traveling wave solution where the solutions $u_{2_{3}}(\xi)$ and $u_{2_{9}}(\xi)$ represent singular solution. The solutions $u_{2_{4}}(\xi)$ express the well-known singular soliton. Figure 5 shows the graphical illustrations of singular soliton $u_{2_{4}}(\xi)$ in (3.31) for $\mu=-2, \sigma=-1, \xi_{0}=2, a_{0}=3, k=1, \alpha=1, \beta=2, \lambda=0$ within the interval $-5 \leq x \leq 5$ and $-3 \leq t \leq 3$. The Singular kink wave solution $u_{27}(\xi)$ in (3.34) for $\mu=-2, \sigma=-1, \xi_{0}=1, a_{0}=3, k=1, \alpha=1, \beta=2, \lambda=1$ within the interval $-5 \leq x, t \leq 5$ is given in Figure 6 . For simplicity we ignored the others figures.

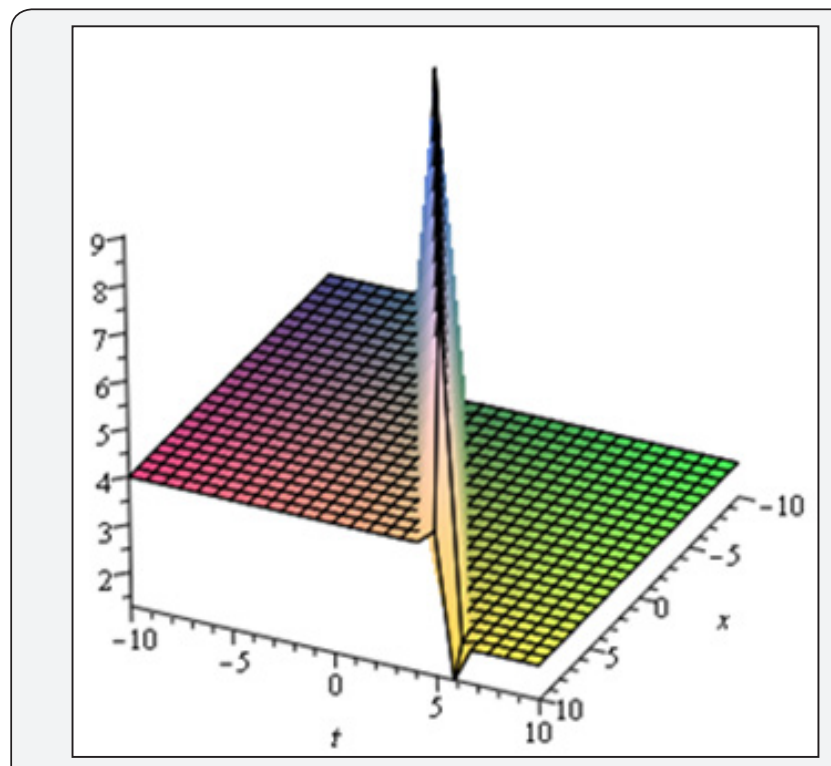

Figure 2: Singular kink wave solution $u_{s}(\xi)$ in (3.9) for $\mu=-1, \sigma=1, \xi_{0}=1, a_{0}=3, \lambda=2$.

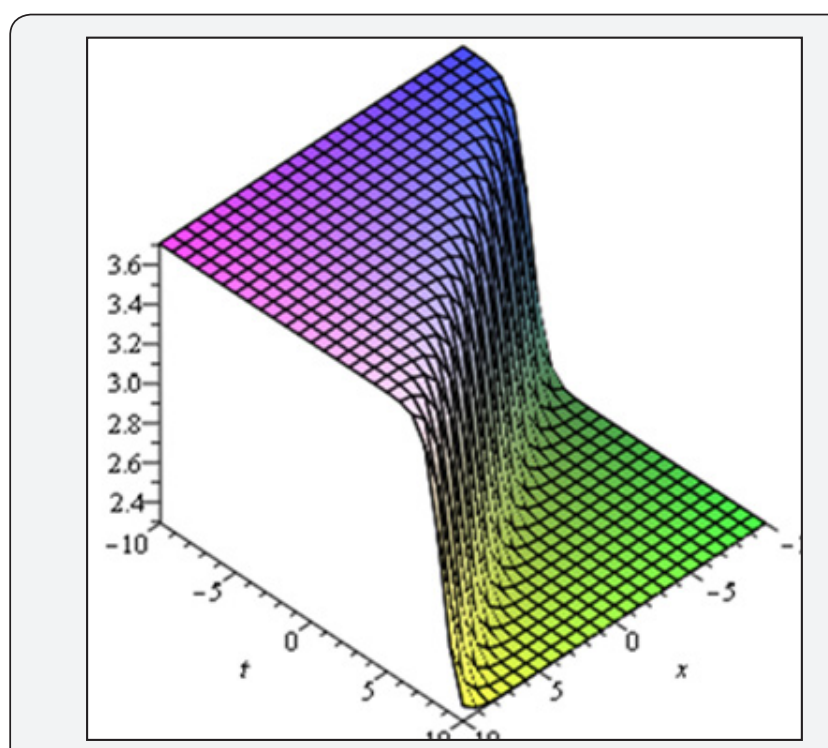

Figure 3: Kink shape wave solution $u_{8}(\xi)$ in (3.12) for $\mu=-2, \sigma=1, \xi_{0}=3, a_{0}=2, \lambda=1$. 


\section{Biostatistics and Biometrics Open Access Journal}

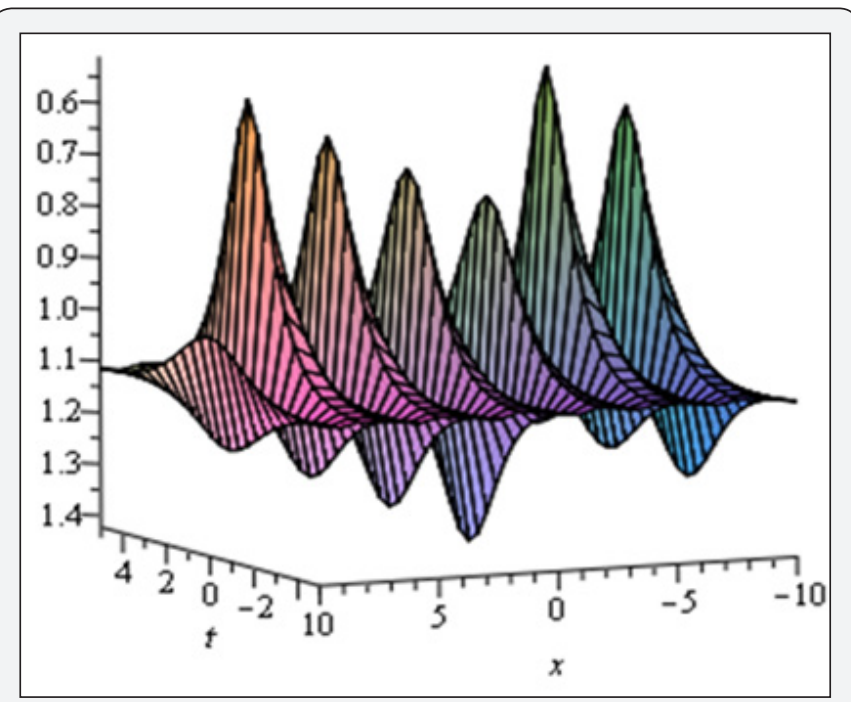

Figure 4: Periodic wave solution $u_{19}(\xi)$ in (3.23) for $\mu=1, \sigma=1, \xi_{0}=1, a_{0}=2, \lambda=1$.

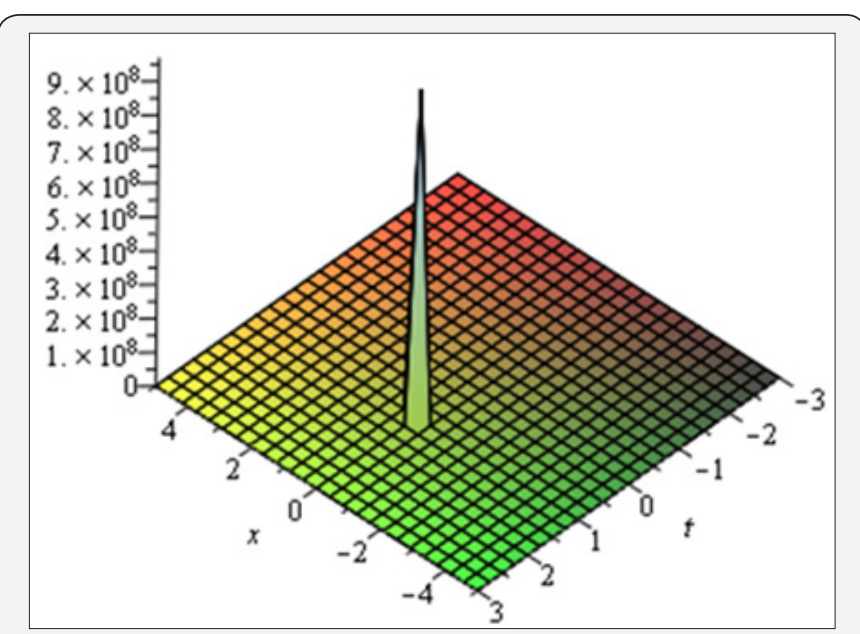

Figure 5: Soliton solution $u_{2_{4}}(\xi)$ in (3.31) for $\mu=-2, \sigma=-1, \xi_{0}=2, a_{0}=3, k=1, \beta=2, \lambda=0$.

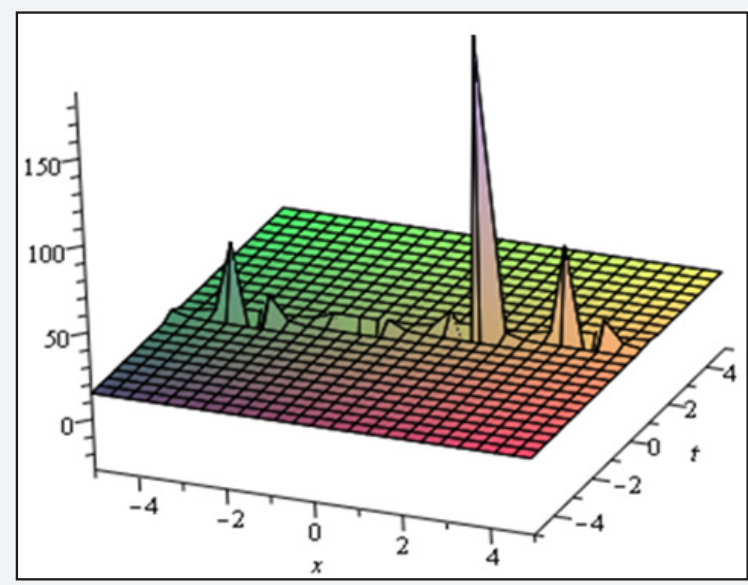

Figure 6: Singular kink solution $u_{(27)}(\xi)$ in (3.34) for $u_{2,}(\xi) \mu=-1, \sigma=-1, \xi_{0}=1, a_{0}=3, k=1, \beta=2, \lambda=1$.

\section{Conclusion}

In this article, enhanced $\left(G^{\prime} / G\right)$-expansion method has been successfully applied to find the closed form wave solutions of new fifth order nonlinear equation and new generalized fifth order nonlinear equation. The solutions are verified to check the correctness of the solutions by putting them back into the original equation and found correct. The key advantage of the enhanced $\left(G^{\prime} / G\right)$-expansion method against other methods is that the method provides more general and huge amount of new closed form wave solutions. The closed form solutions have its great importance to interpretation the inner mechanism of the complex physical phenomena. Therefore this method is very concise and straightforward to handling and can be applied for finding closed form solutions of other NLEEs arising in science and engineering.

\section{References}

1. Yang L, Liu J, Yang K (2001) Exact solutions of nonlinear PDE nonlinear transformation and reduction of nonlinear PDE to a quadrature. Phys Lett A 278: 267-270.

2. Taghizadeh N, Mirzazadeh M (2011) The first integral method to some complex nonlinear partial differential equations, J.Comput. Appl Math 235: 4871-4877.

3. Sirendaoreji (2004) New exact travelling wave solutions for the Kawahara and modified Kawahara equations, Chaos Solitons Fract 19: 147-150.

4. Bekir $A B$ (2008) Exact solutions for nonlinear evolution equations using Exp-function method. Phy Lett A 372: 1619-1625.

5. Akbar MA, Ali NHM (2011) Exp-function method for Duffing equation and new solutions of $(2+1)$ dimensional dispersive long wave equations. Prog Appl Math 1(2).

6. Naher H, Abdullah AF, Akbar MA (2011) The Exp-function method for new exact solutions of the nonlinear partial differential equations. Int J Phys Sci 6(29): 6706-6716.

7. Chen Y, Wang Q (2005) Extended Jacobi elliptic function rational expansion method and abundant families of Jacobi elliptic function solutions to the $(1+1)$-dimensional nonlinear dispersive long wave equation. Chaos Solitons \& Fractals 24: 745-757.

8. Liu S, Fu Z, Liu SD, Zhao Q (2001) Jacobi elliptic function expansion method and periodic wave solutions of nonlinear equations. Phys Lett A 289: 69-74.

9. Priya NV, Senthilvelan M (2015) Generalized Darboux transformation and $\mathrm{N}$-th order rogue wave solution of a general coupled nonlinear schrodinger equations. commum Sci Numer Simul 20: 401-420.

10. Chow KW (1995) A class of exact periodic solutions of nonlinear envelope equation. J Math Phys 36: 4125-4137.

11. Wang ML, Zhou YB (2003) The periodic wave equations for the Klein Gordon Schordinger equations. Phys Lett A 318: 84-92.

12. Adomian G (1994) Solving Frontier Problems of Physics: The Decomposition Method. Kluwer Academic Publishers, Boston, MA, USA.

13. Cevikel AC, Beker A, Akar M, San S (2012) A procedure to construct exact solution of nonlinear evolution equations. Pramana J Phys 79(3): 337-344.

14. Wazwaz AM (2004) A Applied sine-cosine method for handle nonlinear wave equations. Math Comput Modeling 40: 499-508. 
15. Weiss J, Tabor M, Carnevale G (1982) The Painlevé property for partial differential equations. J Math Phys 24(3): 522-526.

16. Khan K, Akbar MA (2013) Application of $\exp (-\Phi(\eta))$-expansion method to find the exact solutions of modified Benjamin-Bona-Mahony equation. World Appl Sci J 24(10): 1373-1377.

17. Islam R, Alam MN, Hossain AKMKS, Roshid HO, Akbar MA (2013) Traveling wave solutions of nonlinear evolution equations via Exp $(-\Phi(\eta))$-expansion method. Global J Sci Frontier Res 13(11): 63-71.

18. Helal MA, Seadawy AR (2009) Variational method for the derivative nonlinear Schrodinger equation with computational applications. Physica Scripta 80: 350-360.

19. Seadawy AR (2011) New exact solutions for the KdV equation with higher order nonlinearity by using the variational method. Comp and Math Appl 62: 3741-3755.

20. Wazwaz AM (2014) kink solutions for three new fifth order nonlinear equations. App Math Modeling 38(1): 110-118.

21. Wazwaz AM (2004) A Applied sine-cosine method for handle nonlinear wave equations. Math Comput Modeling 40: 499-508.

22. Lee J, Sakthival R (2013) New exact travelling wave solutions for some important nonlinear physical models. Pramana J phys 80(5): 757-769.

23. Seadawy AR (2014) Stability analysis for Zakharov-Kuznetsov equation of weakly nonlinear ion-acoustic waves in a plasma. Comp and Math Appl 67(1): 172-180.

24. Seadawy AR (2016) Three-dimensional nonlinear modified ZakharovKuznetsov equation of ion-acoustic waves in a magnetized plasma. Comp and Math Appl 71(1): 201-212.

25. Akter J, Akbar MA (2015) Exact solutions to the Benney-Luke equation and the Phi- 4 equations by using modified simple equation method. Results in phys 5: 125-130.

26. Khan K, Akbar MA (2013) Exact and solitary wave solutions for the Tzitzeica-Dodd-Bullough and the modified KdV-Zakharov-Kuznetsov equations using the modified simple equation method. Ain Shams Eng J 4(4): 903-909.

27. Hossain AKMKS, Akbar MA, Wazwaz AM (2017) Closed form solutions of complex wave equations via modified simple equation method. Cogent Phys 4: 1312751.
28. Khan K, Akbar MA, Ali NHM (2013) The Modified simple equation method for exact and solitary wave solutions of nonlinear evolution equation: the GZK-BBM equation and right-handed non-commutative burgers equations. ISRN Math Phys p. 5.

29. Hossain AKMKS, Akbar MA (2017) Traveling wave solutions of nonlinear evolution equations via Modified simple equation method. Int J of App Math and theoretical Phys 3(2): 20-25.

30. Guo AL, Lin J (2010) Exact solutions of (2+1)-dimensional HNLS equation. Commun Theor Phys 54: 401-406.

31. Akbar MA, Ali NHM, Zayed EME (2012) Abundant exact traveling wave solutions of the generalized Bretherton equation via $\left(G^{\prime} / G\right)$-expansion method. Commun Theor Phys 57(2): 173-178.

32. Alam MN, Akbar MA, Roshid HO (2013) Study of nonlinear evolution equations to construct traveling wave solutions via the new approach of generalized $\left(G^{\prime} / G\right)$ - expansion method. Math Stat 1(3): 102-112.

33. Naher H, Abdullah FA (2014) New Approach of (G'/G)-expansion Method for RLW Equation. Res J of App Sci Eng and Tech 7(23): 48644871.

34. Akbar MA, Ali NHM, Din STM (2012) The alternative (G'/G)-expansion method with generalized Riccati equation: application to fifth order (1+1)-dimensional Caudrey-Dodd-Gibbon equation. Int J Phys Sci 7(5): 743-752.

35. Manafianheris J (2012) Exact solutions of the BBM and MBBM equations by the generalized $\left(G^{\prime} / G\right)$ - expansion method. Int J Genetic Engr 2(3): 28-32.

36. Khan K, Akbar MA (2014) Traveling wave solutions of nonlinear evolution equations via the enhanced $\left(G^{\wedge^{\prime}} / G\right)$-expansion method. Egypt Math Soc 22(2): 220-226.

37. Hossain AKMKS, Akbar MA (2017) Closed form solutions of two nonlinear equations via enhanced $\left(\mathrm{G}^{\wedge^{\prime}} / \mathrm{G}\right)$-expansion method. Cogent Math 4(1): 1312751.

38. H Naher, Abdullah FA (2014) The improved (G'/G)-expansion method to the $(2+1)$-dimensional breaking soliton equation. Journal of Computational Analysis \& Applications 16(2): 220-235.

\section{Your next submission with Juniper Publishers}

will reach you the below assets

- Quality Editorial service

- Swift Peer Review

- Reprints availability

- E-prints Service

- Manuscript Podcast for convenient understanding

- Global attainment for your research

- Manuscript accessibility in different formats

( Pdf, E-pub, Full Text, Audio)

- Unceasing customer service

Track the below URL for one-step submission https://juniperpublishers.com/online-submission.php 